\title{
Combinatorial Biomaterials Discovery Strategy to Identify New Macromolecular Cryoprotectants
}

\author{
Christopher Stubbs, Kathryn A. Murray, Toru Ishibe, Robert T. Mathers, and Matthew I. Gibson*
}

Cite This: ACS Macro Lett. 2020, 9, 290-294

Read Online

ABSTRACT: Cryoprotective agents (CPAs) are typically solvents or small molecules, but there is a need for innovative CPAs to reduce toxicity and increase cell yield, for the banking and transport of cells. Here we use a photochemical high-throughput discovery platform to identify macromolecular cryoprotectants, as rational design approaches are currently limited by the lack of structure-property relationships. Using liquid handling systems,

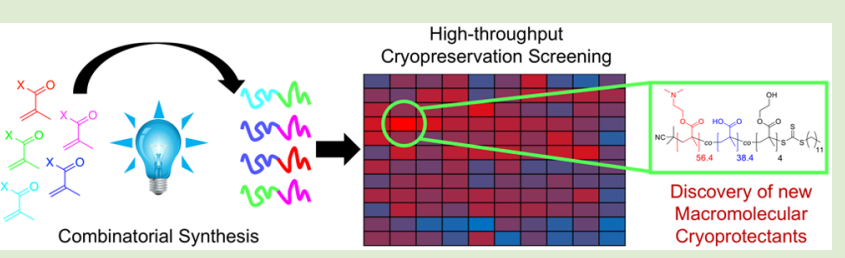
120 unique polyampholytes were synthesized using photopolymerization with RAFT agents. Cryopreservation screening identified "hit" polymers and nonlinear trends between composition and function, highlighting the requirement for screening, with polymer aggregation being a key factor. The most active polymers reduced the volume of dimethyl sulfoxide (DMSO) required to cryopreserve a nucleated cell line, demonstrating the potential of this approach to identify materials for cell storage and transport.

$\mathrm{T}$ he distribution of cells as therapies, as protein factories, ${ }^{1-3}$ and in basic biomedical research is underpinned by their cryopreservation to enable storage and distribution. This is essential as cells cannot be maintained in continuous culture due to the resulting genetic and phenotypic drift. ${ }^{4}$ Current cryopreservation protocols for mammalian cells rely on the addition of high concentrations of dimethyl sulfoxide (DMSO) as the cryoprotective agent (CPA). While widely used, DMSO does not give full recovery of all cells post-thaw (leading to wastage) and is intrinsically cytotoxic (leading to further cell death if left in contact)..$^{5-7}$ DMSO does not protect against all mechanisms of cell death (e.g., mechanical damage caused by ice recrystallization ${ }^{8}$ ). It is therefore desirable to reduce the amount of DMSO used in cryoprotective solutions. To address this issue, macromolecular cryoprotectants inspired by antifreeze (glyco) proteins or late embryogenesis abundant proteins are emerging. ${ }^{9-11}$ Polymers which control ice recrystallization have been found to give some benefit during cryopreservation of various cell lines, but this effect is limited in mammalian cells. ${ }^{12}$ However, it is emerging that polyampholytes (polymers with a balance of cationic and anionic side chains) are extremely potent cryopreservation enhancers despite only having moderate ice recrystallization inhibition (IRI) activity ${ }^{13,14}$ compared to, e.g., poly(vinyl alcohol) or other inhibitors. ${ }^{15-17}$ Polyampholytes have been shown to be remarkably potent cryoprotectants for many cell types including mesenchymal stem cell (MSC) monolayers, ${ }^{18}$ chondrocyte sheets, ${ }^{19}$ and human MSCs. ${ }^{20}$ However, their mode of action remains unclear, in part due to the lack of structure-property relationships. There is some evidence that polyampholytes engage and protect cell membranes, but this is not proven as their mode of cryoprotection. $^{14,18}$
In any biomimetic material, a key challenge is the exploration of sufficiently large chemical space (100s of materials) to enable key structural motifs to be identified. This is a particular challenge in macromolecular cryoprotectants due to their diverse modes of action and paucity of published structures of active materials. Alexander and coworkers have used microarray printing and UV-photocuring to explore 1000 s of copolymers to identity surfaces suitable for resisting bacterial adhesion and for the expansion of stem cells. $^{21}$ Schubert and co-workers exploited liquid handling systems for automated cationic and radical polymerizations. ${ }^{22}$ However, this required significant infrastructure and robust handling methods to exclude oxygen, which prematurely terminates radical polymerizations. Recently, there has been a revolution in oxygen-tolerant controlled radical polymerization methods, ${ }^{23}$ for example, protein $^{24}$ or tertiary amine degassing, ${ }^{25}$ breathing ATRP, ${ }^{26}$ and PET-RAFT. ${ }^{27}$ A benefit of these methods is that little infrastructure is required to conduct the reactions in industry-standard multiwell plates; almost all biological testing is conducted in 96-well plates. Richards et al. utilized blue-light-initiated open-air RAFT photopolymerization to identify new antimicrobial polymers. ${ }^{28}$ Chapman and co-workers used oxygen-tolerant PET-RAFT to make a library of 18 lectin binding materials. ${ }^{27}$ There are currently no detailed structure-activity relationships in the field of macromolecular cryoprotectants which is preventing the rational design of new materials.

Received: January 17, 2020

Accepted: February 6, 2020

Published: February 7, 2020 
This manuscript describes the first biomaterials discovery approach to identify macromolecular cryoprotectants. Using liquid-handling systems and photo-RAFT polymerization, a library of polymers were synthesized, characterized, and screened for cryopreservation. A new cryoprotectant terpolymer was discovered which enabled nucleated cell cryopreservation with reduced [DMSO].

2-(Dimethylamino)ethyl methacrylate (DMEAMA) and methacrylic acid (MAA) were selected as the cationic/anionic components based on previous reports. ${ }^{14,29}$ Initial screening (Supporting Information) identified that an excess of DMEAMA compared to MAA leads to improved cryopreservation in an erythrocyte model, so a 6:4 DMEAMA:MAA ratio was used. To enable high-throughput polymer synthesis, liquid-handling robots were used to distribute reagents within 96-well plates, which is also the format for the cryopreservation testing. Blue-light-mediated polymerization using a trithiocarbonate and triethanolamine (TEOA) as the degassing agent was employed (Figure 1A). ${ }^{25,30}$ [Controls on the role of TEOA are in Figures S4/5]. To tune the polyampholyte, a panel of 12 (uncharged) comonomers were selected (Figure 1B). These were distributed by the liquid-handling system at 2-20 mol \% with DMEAMA/MAA. An amount of $20 \mathrm{~mol} \%$ was the upper limit to ensure solubility of the library. Polymerizations were conducted in 96-well plates under blue-light irradiation and then dried under a vacuum. [Note this method gives larger dispersities than a true CRP process. ${ }^{31}$ ] A fraction was removed for size exclusion chromatography (SEC), revealing monomodal distributions and reproducible molecular weights within each polymer class (Figure 1B and Table S2).

Erythrocytes (red blood cells) are a useful model system for cryopreservation, as when damaged, heamoglobin leaks, which can be measured by the alkaline hemeatin detergent (AHD) assay, suitable for plate-based analysis. ${ }^{32}$ A sample of polymer from the polymerization plate (at $140 \mathrm{mg} \cdot \mathrm{mL}^{-1}$ in $700 \mu \mathrm{L}$ of PBS) was transferred directly into the corresponding wells of a 96-well plate and diluted to $71 \mathrm{mg} \cdot \mathrm{mL}^{-1}$ by the addition of red blood cells suspended in PBS. This mixture was then frozen in liquid nitrogen vapor and thawed at $45^{\circ} \mathrm{C}$, and any intact cells and debris were removed by centrifugation of the plate. The supernatant was sampled into AHD solutions and hemolysis measured on a plate reader at $580 \mathrm{~nm}$. All 120 polymers were assessed for cryopreservation capability in triplicate, and the results are summarized in the heat map shown in Figure $2 \mathrm{~B}$.

Figure $2 \mathrm{~B}$ reveals the essential need for high-throughput screening in this emerging field; nonlinear relationships between mol \% comonomer and recovery were observed, meaning hits would be missed when using traditional small panels of polymers. For example, using comonomer 1 (PEG methacrylate), at 8 or $14 \mathrm{~mol} \%$, led to increased recovery, compared to $10 \mathrm{~mol} \%$ which led to a significant reduction-a feature which could easily be missed in targeted synthesis. Octanol/water partition coefficients, normalized to surface area $(\log P / S A)$, were calculated using oligomers of the comonomers and reported in Figure $2 \mathrm{~B},{ }^{3,34}$ revealing no clear correlation. This demonstrated that any assumptions of "more hydrophobic is better" are oversimplified and limited by the current lack of understanding of how polyampholytes function. ${ }^{18,35}$ This should be appropriately described as hydrophobicity is beneficial to a point and should be experimentally verified. ${ }^{18}$ The most active polymer identified contained $4 \mathrm{~mol} \%$ of hydroxyethyl methacrylate (4), with
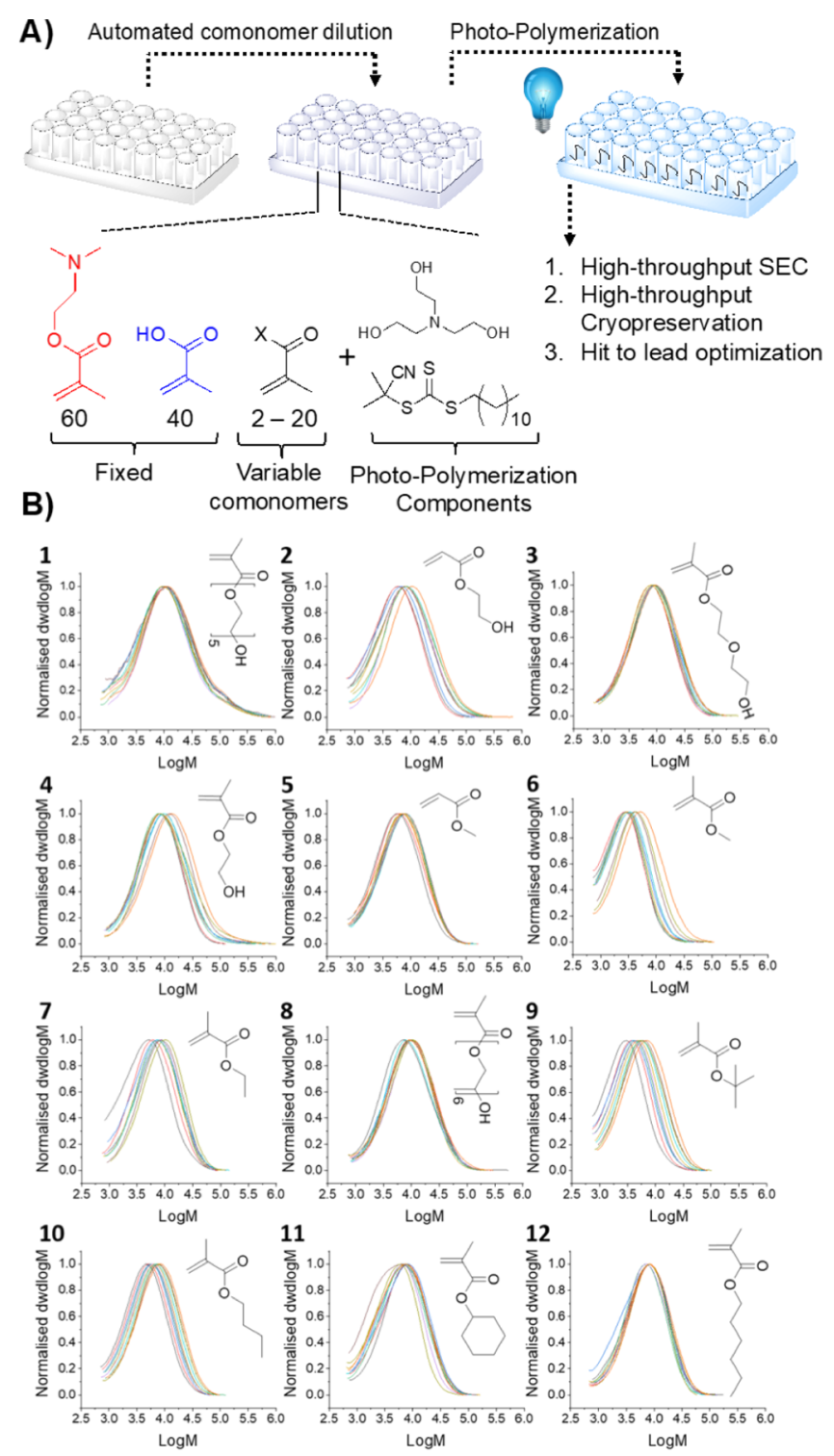

Co-monomer $\mathrm{mol} \%$

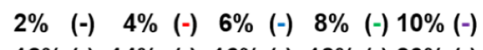

$12 \%(-) 14 \%(-) 16 \%(-) 18 \%(-) 20 \%(-)$

Figure 1. (A) Combinatorial photopolymerization strategy employed here. (B) SEC analysis of the polymer library. Number indicates comonomer used. Polymers were synthesized at a $[\mathrm{M}]:[\mathrm{CTA}]$ ratio of 100:1.

other levels of incorporation also giving significant cell recovery. In contrast, the excessive hydrophobicity of cyclohexyl methacrylate (11) resulted in the worst performance.

The best and worst polymers from the cryopreservation screen (4\% HEMA and 10\% CyMA, respectively) were taken forward to test in a nucleated cell model, using well-defined polymers to validate the screening approach. The hit terpolymers were synthesized targeting different DPs, using conventional RAFT polymerization (Table 1 and Figure 3A), and characterized by SEC, NMR, and IR (SI). In an erythrocyte cryopreservation assay, these polymers performed as in the screen (Figure $3 \mathrm{C}$ ), confirming the initial results. Importantly, the hit (4\% HEMA) terpolymer was a better cryoprotectant than the copolymer without additional monomers (Figure S4). 


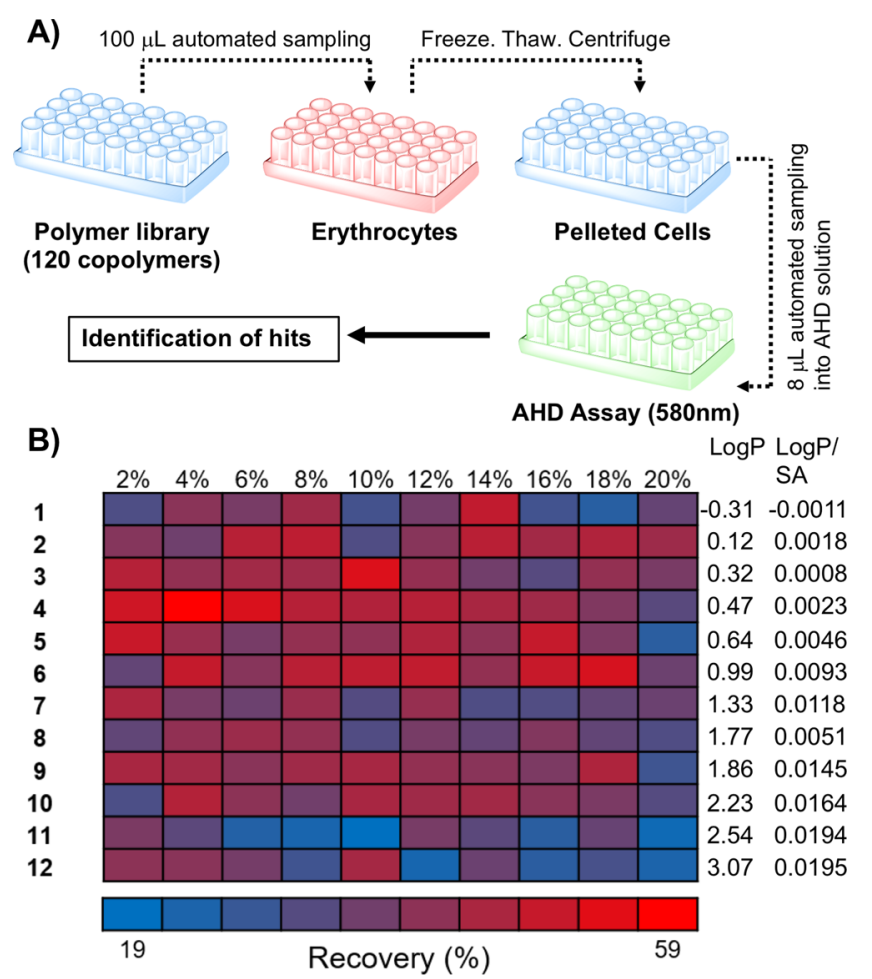

Figure 2. High-throughput screening of cryopreservation against erythrocytes. (A) Schematic of liquid transfer and screening conditions. (B) Heat map showing post-thaw erythrocyte recovery outcomes (recovery $=1$-heamolysis) using $71 \mathrm{mg} \cdot \mathrm{mL}^{-1}$ of polymer. Recoveries are average of three repeats (individual data in SI). Monomer structures are in Figure 1.

Table 1. Hit Polymers Synthesized by RAFT

$\begin{array}{lccccc}\text { polymer }^{a} & \begin{array}{c}{[\mathrm{M}] /[\mathrm{CTA}]^{b}} \\ (-)\end{array} & \begin{array}{c}\text { conversion } \\ (\%)^{c}\end{array} & \begin{array}{c}\mathrm{Mn}_{\left(\mathrm{Theo}^{d}{ }^{d}\right.}^{\left(\mathrm{g} \mathrm{mol}^{-1}\right)} \\ \begin{array}{c}\mathrm{Mn}_{(\mathrm{SEC})^{e}} \\ \left(\mathrm{~g} \mathrm{~mol}^{-1}\right)\end{array}\end{array} & \theta^{e} \\ \begin{array}{l}4 \% \\ \mathrm{HEMA}_{50}\end{array} & 50 & 100 & 6400 & 10000 & 1.48 \\ \begin{array}{l}4 \% \\ \mathrm{HEMA}_{100}\end{array} & 100 & 97 & 12000 & 15000 & 1.57 \\ \begin{array}{l}4 \% \\ \mathrm{HEMA}\end{array} & 200 & 98 & 25000 & 15000 & 1.84 \\ \begin{array}{c}10 \% \\ \mathrm{CyMA}_{100}\end{array} & 100 & 96 & 12000 & 16000 & 1.39 \\ & & & & & \end{array}$

${ }^{a}$ Polymers labeled based on comonomer incorporation ratio into a 6:4 DMEAMA:MA copolymer. ${ }^{b}$ Monomer:CTA ratio. ${ }^{c}$ Determined by ${ }^{1} \mathrm{H}$ NMR. ${ }^{d}$ Based on feed ratio and conversion. ${ }^{e}$ From DMF SEC.

A549 (human Caucasian lung carcinoma) cells were next used as a more challenging model of cryopreservation to assess the hit polymers, selected from the red blood cell cryopreservation screen. A549 cryopreserved in 10 wt \% DMSO yields $>60 \%$ recovery post-thaw. However, exposure of A549s to 10 wt \% of DMSO for just 30 min leads to a $30 \%$ drop in metabolic activity. ${ }^{14}$ We therefore explored cryopreservation using just $2.5 \mathrm{wt} \%$ of DMSO with our polymers added at $20 \mathrm{mg} \cdot \mathrm{mL}^{-1}$ to explore their ability to rescue cell recovery in minimal DMSO. After thawing, the cells were allowed to recover for $24 \mathrm{~h}$ before the total number of viable cells was counted (Figure 3D). [24 h recovery post-thaw removes false positives due to apoptosis. ${ }^{36}$ Copolymers with 4\% HEMA showed enhancements in post-thaw cell recovery increasing from $15 \%$ for DMSO (2.5 wt \%) to above $40 \%$. In contrast, the $10 \%$ CyMA copolymers gave worse results than
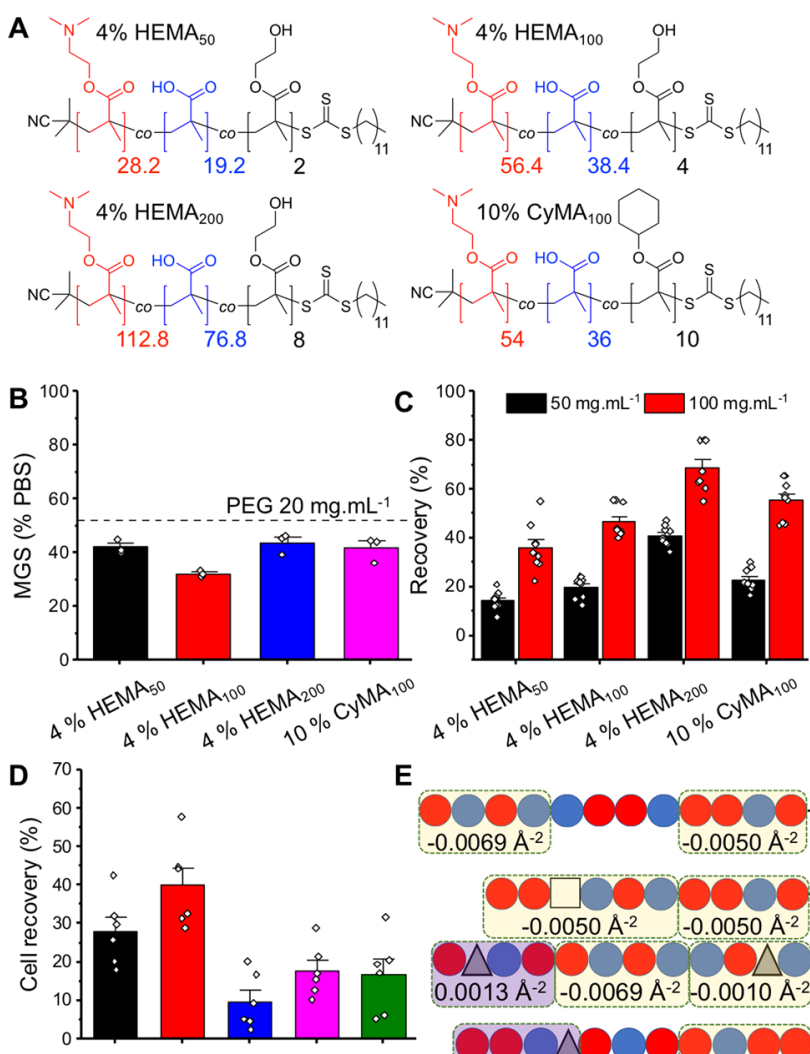

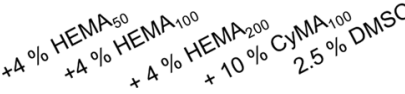

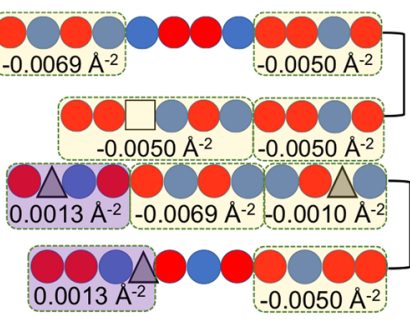

Figure 3. Hit polymers and their cryoprotective properties. (A) Polymers obtained by conventional RAFT polymerization. (B) Ice recrystallization inhibition activity of hit polymers $\left(20 \mathrm{mg} \cdot \mathrm{mL}^{-1}\right.$, MGS = mean grain size, dotted line $=$ PEG $20 \mathrm{mg} \cdot \mathrm{mL}^{-1}$ control). (C) Red blood cell post-thaw recovery. (D) Post-thaw recovery of A549 cells with intact membranes using 2.5 wt $\%$ of $\mathrm{DMSO} / 20 \mathrm{mg} \cdot \mathrm{mL}^{-1}$ of polymer against control of 2.5 wt \% of DMSO alone. (E) Segmented partition coefficient analysis. Colors represent monomers in Part A. Squares are HEMA; triangles are CyMA; and circles are DMAEMA/ MAA. Highlighted areas show net positive or negative $\log P / S A$.

DMSO alone. This shows that polyampholytes are not universal scaffolds and that additional structural features modulate activity. DP100 HEMA was more potent than DP50 or 200 showing that the macromolecular structure is important with the longer polymer performing poorly in this assay. Figure 3E (and S10/11) shows oligomer models of the most/least active copolymers and the segmental LogP/SA values (rather than a crude average across the whole polymer). Localized regions of hydrophobic content (positive log $P / S A$ ) correlated with poor cell recovery, but hydrophilic regions exhibited better performance. This observation may explain why alternating (evenly distributed hydrophobicity) polyampholytes are potent cryopreservatives and also have more IRI activity compared to random. ${ }^{13,14}$ None of the polymers showed significant IRI activity ${ }^{16}$ (Figure $3 \mathrm{~B}$ ) ruling out icegrowth inhibition as a mode of action. ${ }^{37}$ The recoveries obtained here are not as high as a previously reported poly(ampholyte $)^{14}$ but rather show the opportunity to modulate activity and determine the important structural features. Dynamic light scattering (DLS) showed that $10 \%$ CyMA polymers aggregated but that the 4\% HEMA aggregated less. This demonstrates that although hydrophobicity can help, 
aggregation negates the benefits: this could lead to reducing the effective concentrations of the polymers or simply preventing efficient membrane interactions (a hypothesis we highlight is not confirmed).

To further test how the polymers protect cells, carboxyfluorescein-loaded 1,2-dioleoyl-sn-glycero-3- phosphocholine liposomes $(100 \mathrm{~nm})$ were prepared (Supporting Information) to enable evaluation of cryoprotection in a lipid-only system. The most active cellular cryoprotectant ( $4 \%$ HEMA $\left._{100}\right)$ protected liposomes against freezing, equally well as a control polyampholyte (Figure S9). The most hydrophobic polymer $\left(10 \% \mathrm{CyMA}_{100}\right)$ failed to provide protection, which is in contrast to the results of Matsumura et al. ${ }^{18}$ This does not rule out hydrophobicity being beneficial but rather that multiple factors are influencing cryopreservation outcomes including the aggregation state of the materials, and hence a fine balance is required. Antifreeze proteins have been reported to protect membranes and may be a related mechanism of action. ${ }^{38}$ Osmotic effects on cryopreservation due to the use of a polyelectrolyte cannot be ruled out. However, the clear impact of comonomers and chain length on the observed cryopreservation outcomes suggests it is more complex and that a single mechanism may not be responsible.

In summary, we have used a high-throughput discovery platform to identify polyampholyte terpolymers, which are capable of reducing the volume of organic solvents required for cellular cryopreservation. High-throughput oxygen-tolerant photo-RAFT polymerization generated a library of 120 polyampholytes. Screening of this library for red blood cell cryopreservation enabled identification of hits, with terpolymers containing just $4 \mathrm{~mol} \%$ of hydroxyethyl methacrylate showing particularly high activity. "Hit" terpolymers enhanced cryopreservation of a nucleated cell model using just 2.5 wt \% of DMSO, rather than the conventional $10 \mathrm{wt} \%$. The polymers did not function by inhibiting ice growth, but there was some evidence that they can stabilize model membranes. Modeling showed that the most active polymers had negative surfacearea-normalized partition coefficients across most segments. Overall, this study shows that by exploring the chemical space of polyampholytes it may be possible to discover new macromolecular cryoprotectants and access structure-property relationships to help understand how they function.

\section{ASSOCIATED CONTENT}

\section{s) Supporting Information}

The Supporting Information is available free of charge at https://pubs.acs.org/doi/10.1021/acsmacrolett.0c00044.

Experimental details and characterization as well as additional data (PDF)

\section{AUTHOR INFORMATION}

\section{Corresponding Author}

Matthew I. Gibson - Department of Chemistry and Warwick Medical School, University of Warwick, Coventry CV4 7AL, U.K.; (1) orcid.org/0000-0002-8297-1278;

Email: m.i.gibson@warwick.ac.uk

\section{Authors \\ Christopher Stubbs - Department of Chemistry, University of Warwick, Coventry CV4 7AL, U.K. \\ Kathryn A. Murray - Department of Chemistry, University of Warwick, Coventry CV4 7AL, U.K.}

Toru Ishibe - Department of Chemistry, University of Warwick, Coventry CV4 7AL, U.K.

Robert T. Mathers - Department of Chemistry, Pennsylvania State University, University Park, Pennsylvania 15068, United

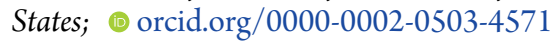

Complete contact information is available at:

https://pubs.acs.org/10.1021/acsmacrolett.0c00044

\section{Notes}

The authors declare no competing financial interest.

\section{ACKNOWLEDGMENTS}

MIG holds ERC grants (638661 and 789182). The JSR Corporation is thanked for financial support of T.I. and The MRC (MC_PC_17203) for supporting K.M. The Polymer $\mathrm{RTP}$ at $\mathrm{UoW}$ is thanked for support with SEC analysis.

\section{REFERENCES}

(1) Swartz, M. A.; Hirosue, S.; Hubbell, J. A. Engineering Approaches to Immunotherapy. Sci. Transl. Med. 2012, 4 (148), 148 rv9.

(2) Healy, K. E.; McDevitt, T. C.; Murphy, W. L.; Nerem, R. M. Engineering the Emergence of Stem Cell Therapeutics. Sci. Transl. Med. 2013, 5 (207), 207ed17.

(3) Levine, B. L.; Miskin, J.; Wonnacott, K.; Keir, C. Global Manufacturing of CAR T Cell Therapy. Mol. Ther.-Methods Clin. Dev. 2017, 4, 92-101.

(4) Torsvik, A.; Stieber, D.; Enger, P. O.; Golebiewska, A.; Molven, A.; Svendsen, A.; Westermark, B.; Niclou, S. P.; Olsen, T. K.; Chekenya Enger, M.; Bjerkvig, R. U-251 Revisited: Genetic Drift and Phenotypic Consequences of Long-Term Cultures of Glioblastoma Cells. Cancer Med. 2014, 3 (4), 812-824.

(5) Zambelli, A.; Poggi, G.; Da Prada, G. A.; Pedrazzoli, P.; Cuomo, A.; Miotti, D.; Perotti, C.; Preti, P.; Cuna, G. R. Clinical Toxicity of Cryopreserved Circulating Progenitor Cells Infusion. Anticancer Res. 1998, 18 (6B), 4705-4708.

(6) Kawai, K.; Li, Y. S.; Song, M. F.; Kasai, H. DNA Methylation by Dimethyl Sulfoxide and Methionine Sulfoxide Triggered by Hydroxyl Radical and Implications for Epigenetic Modifications. Bioorg. Med. Chem. Lett. 2010, 20 (1), 260-265.

(7) Timm, M.; Saaby, L.; Moesby, L.; Hansen, E. W. Considerations Regarding Use of Solvents in in Vitro Cell Based Assays. Cytotechnology 2013, 65 (5), 887-894.

(8) Mazur, P. Freezing of Living Cells: Mechanisms and Implications. Am. J. Physiol. Physiol. 1984, 247 (3), C125-C142.

(9) Biggs, C. I.; Bailey, T. L.; Stubbs, C.; Fayter, A.; Gibson, M. I. Polymer Mimics of Biomacromolecular Antifreezes. Nat. Commun. 2017, 8 (1), 1546

(10) Balcerzak, A. K.; Capicciotti, C. J.; Briard, J. G.; Ben, R. N. Designing Ice Recrystallization Inhibitors: From Antifreeze (Glyco)Proteins to Small Molecules. RSC Adv. 2014, 4 (80), 42682-42696.

(11) He, Z.; Liu, K.; Wang, J. Bioinspired Materials for Controlling Ice Nucleation, Growth, and Recrystallization. Acc. Chem. Res. 2018, 51 (5), 1082-1091.

(12) Chao, H.; Davies, P. L.; Carpenter, J. F. Effects of Antifreeze Proteins on Red Blood Cell Survival during Cryopreservation. J. Exp. Biol. 1996, 199, 2071-2076.

(13) Stubbs, C.; Lipecki, J.; Gibson, M. I. Regioregular Alternating Polyampholytes Have Enhanced Biomimetic Ice Recrystallization Activity Compared to Random Copolymers and the Role of Side Chain versus Main Chain Hydrophobicity. Biomacromolecules 2017, 18 (1), 295-302.

(14) Bailey, T. L.; Stubbs, C.; Murray, K.; Tomas, R. M. F.; Otten, L.; Gibson, M. I. A Synthetically Scalable Poly(Ampholyte) Which Dramatically Enhances Cellular Cryopreservation. Biomacromolecules 2019, 20, 3104-3114. 
(15) Congdon, T.; Notman, R.; Gibson, M. I. Antifreeze (Glyco)Protein Mimetic Behavior of Poly(Vinyl Alcohol): Detailed Structure Ice Recrystallization Inhibition Activity Study. Biomacromolecules 2013, 14 (5), 1578-1586.

(16) Biggs, C. I.; Stubbs, C.; Graham, B.; Fayter, A. E. R.; Hasan, M.; Gibson, M. I. Mimicking the Ice Recrystallization Activity of Biological Antifreezes. When Is a New Polymer "Active"? Macromol. Biosci. 2019, 19 (7), 1900082.

(17) Drori, R.; Li, C.; Hu, C.; Raiteri, P.; Rohl, A. L.; Ward, M. D.; Kahr, B. A Supramolecular Ice Growth Inhibitor. J. Am. Chem. Soc. 2016, 138 (40), 13396-13401.

(18) Rajan, R.; Hayashi, F.; Nagashima, T.; Matsumura, K. Toward a Molecular Understanding of the Mechanism of Cryopreservation by Polyampholytes: Cell Membrane Interactions and Hydrophobicity. Biomacromolecules 2016, 17 (5), 1882-1893.

(19) Maehara, M.; Sato, M.; Watanabe, M.; Matsunari, H.; Kokubo, M.; Kanai, T.; Sato, M.; Matsumura, K.; Hyon, S. H.; Yokoyama, M.; Mochida, J.; Nagashima, H. Development of a Novel Vitrification Method for Chondrocyte Sheets. BMC Biotechnol. 2013, 13 (1), 58.

(20) Matsumura, K.; Hayashi, F.; Nagashima, T.; Hyon, S. H. LongTerm Cryopreservation of Human Mesenchymal Stem Cells Using Carboxylated Poly-l-Lysine without the Addition of Proteins or Dimethyl Sulfoxide. J. Biomater. Sci., Polym. Ed. 2013, 24 (12), 14841497.

(21) Hook, A. L.; Chang, C. Y.; Yang, J.; Luckett, J.; Cockayne, A.; Atkinson, S.; Mei, Y.; Bayston, R.; Irvine, D. J.; Langer, R.; Anderson, D. G.; Williams, P.; Davies, M. C.; Alexander, M. R. Combinatorial Discovery of Polymers Resistant to Bacterial Attachment. Nat. Biotechnol. 2012, 30 (9), 868-875.

(22) Hoogenboom, R.; Fijten, M. W. M.; Meier, M. A. R.; Schubert, U. S. Macromol. Rapid Commun.. 2003, 24, 92-97.

(23) Oliver, S.; Zhao, L.; Gormley, A. J.; Chapman, R.; Boyer, C. Living in the Fast Lane - High Throughput Controlled/Living Radical Polymerization. Macromolecules 2019, 52, 3-23.

(24) Chapman, R.; Gormley, A. J.; Herpoldt, K.-L.; Stevens, M. M. Highly Controlled Open Vessel RAFT Polymerizations by Enzyme Degassing. Macromolecules 2014, 47 (24), 8541-8547.

(25) Fu, Q.; Xie, K.; McKenzie, T. G.; Qiao, G. G. Trithiocarbonates as Intrinsic Photoredox Catalysts and RAFT Agents for Oxygen Tolerant Controlled Radical Polymerization. Polym. Chem. 2017, 8 (9), 1519-1526.

(26) Enciso, A. E.; Fu, L.; Russell, A. J.; Matyjaszewski, K. A Breathing Atom-Transfer Radical Polymerization: Fully OxygenTolerant Polymerization Inspired by Aerobic Respiration of Cells. Angew. Chem., Int. Ed. 2018, 57, 933-936.

(27) Gormley, A. J.; Yeow, J.; Ng, G.; Conway, Ó.; Boyer, C.; Chapman, R. An Oxygen-Tolerant PET-RAFT Polymerization for Screening Structure-Activity Relationships. Angew. Chem., Int. Ed. 2018, 57 (6), 1557-1562.

(28) Richards, S.-J.; Jones, A.; Tomás, R. M. F.; Gibson, M. I. Photochemical "In-Air" Combinatorial Discovery of Antimicrobial Co-Polymers. Chem. - Eur. J. 2018, 24 (52), 13758-13761.

(29) Rajan, R.; Jain, M.; Matsumura, K. Cryoprotective Properties of Completely Synthetic Polyampholytes via Reversible AdditionFragmentation Chain Transfer (RAFT) Polymerization and the Effects of Hydrophobicity. J. Biomater. Sci., Polym. Ed. 2013, 24 (15), $1767-1780$.

(30) Richards, S.-J.; Jones, A.; Tomás, R. M.; Gibson, M. I. Photochemical "In-Air" Combinatorial Discovery of Antimicrobial Copolymers. Chem. - Eur. J. 2018, 24 (52), 13758-13761.

(31) Stubbs, C.; Congdon, T.; Davis, J.; Lester, D.; Richards, S.-J.; Gibson, M. I. High-Throughput Tertiary Amine Deoxygenated Photopolymerizations for Synthesizing Polymer Libraries. Macromolecules 2019, 52, 7603-7612.

(32) Heuck, C. C.; Reinauer, H.; Wood, W. G. The Alkaline Haematin Detergent (AHD575) Method for the Determination of Haemoglobin in Blood-a Candidate Reference Measurement Procedure. Clin. Lab. 2008, 54 (7-8), 255-272.
(33) Foster, J. C.; Varlas, S.; Couturaud, B.; Jones, J. R.; Keogh, R.; Mathers, R. T.; O’Reilly, R. K. Predicting Monomers for Use in Polymerization-Induced Self-Assembly. Angew. Chem., Int. Ed. 2018, 57 (48), 15733-15737.

(34) Dharmaratne, N. U.; Jouaneh, T. M. M.; Kiesewetter, M. K.; Mathers, R. T. Quantitative Measurements of Polymer Hydrophobicity Based on Functional Group Identity and Oligomer Length. Macromolecules 2018, 51 (21), 8461-8468.

(35) Stubbs, C.; Bailey, T. L.; Murray, K.; Gibson, M. I. Polyampholytes as Emerging Macromolecular Cryoprotectants. Biomacromolecules 2020, 21, 7.

(36) Baust, J. M.; Vogel, M. J.; Van Buskirk, R.; Baust, J. G. A Molecular Basis of Cryopreservation Failure and Its Modulation to Improve Cell Survival. Cell Transplant 2001, 10 (7), 561-571.

(37) Deller, R. C.; Vatish, M.; Mitchell, D. A.; Gibson, M. I. Synthetic Polymers Enable Non-Vitreous Cellular Cryopreservation by Reducing Ice Crystal Growth during Thawing. Nat. Commun. 2014, 5, 3244.

(38) Tomczak, M. M.; Hincha, D. K.; Estrada, S. D.; Feeney, R. E.; Crowe, J. H. Antifreeze Proteins Differentially Affect Model Membranes during Freezing. Biochim. Biophys. Acta, Biomembr. 2001, 1511 (2), 255-263. 